\title{
Clinical and pathological response to neoadjuvant chemotherapy with different chemotherapy regimens predicts the outcome of locally advanced breast cancer
}

\author{
Shicong Tang", Ke Wang", Kai Zheng, Jiadong Liu, Hengyu Zhang, Mingjian Tan, Hongwan Li, \\ Huimeng Li, Xin Tan, Dequan Liu, Rong Guo \\ Department of Breast Surgery, the Third Affiliated Hospital of Kunming Medical University, Yunnan Cancer Hospital, Kunming, China \\ Contributions: (I) Conception and design: R Guo; (II) Administrative support: D Liu, K Zheng, J Liu; (III) Provision of study materials or patients: H \\ Li, M Tan, H Zhang; (IV) Collection and assembly of data: K Wang; (V) Data analysis and interpretation: K Wang, S Tang; (VI) Manuscript writing: \\ All authors; (VII) Final approval of manuscript: All authors. \\ \#These authors contributed equally to this work. \\ Correspondence to: Dequan Liu; Rong Guo. No. 519th, Kunzhou Road, Xishan District, Kunming, China. Email: liu_dequan2018@126.com; \\ guorong2320@126.com.
}

Background: This retrospective analysis was designed to research whether clinical response partial response (PR)/complete response (CR) and pathological response (PCR) to neoadjuvant chemotherapy can translate into prognosis benefit pathological response in patients with locally advanced breast cancer and whether different chemotherapy regimens will influence the outcomes.

Methods: One hundred and thirty-five patients with breast cancer patients who received neoadjuvant chemotherapy were included in the retrospective analysis. Patients were followed up strictly. Overall survival (OS) was evaluated by the Kaplan-Meier analysis. The comparison of the clinical and pathological characteristics and recurrence was performed using the carried out by chi-squared and Fisher's exact tests. Univariate and multivariate analyses were performed by the Cox regression analysis.

Results: Clinical response was strongly correlated with lymph nodes status $(\mathrm{P}=0.032)$. The OS comparison of pathological response between the pCR group and non-pCR groups did not exhibit statistically significant differences $(\mathrm{P}=0.400)$. A similar non-significant response result was observed in the comparison of clinical response between the $\mathrm{PR} / \mathrm{CR}$ and $\mathrm{SD} / \mathrm{PD}$ groups group $(\mathrm{P}=0.108)$. Univariate and multivariate analyses did not support clinical response $(\mathrm{P}=0.156 \mathrm{P}=0.095$ respectively) or pathological response $(\mathrm{P}=0.600$ $\mathrm{P}=0.144$ respectively) as the predictors of prognosis. There were no significant differences in either the comparison of the clinical response group it seems no statistically significance $(\mathrm{P}=0.496)$ or the comparison of the pathological response group $(\mathrm{P}=0.460)$. OS analyses across different neoadjuvant chemotherapy regimens demonstrated no significant differences $(\mathrm{P}=0.307)$. In the $\mathrm{PR} / \mathrm{CR}$ and $\mathrm{PD} / \mathrm{SD}$ comparison of every single regimen, there were no significant differences. However, for patients with PR/CR patients from the comparison of five regimens, namely, TAC, FAC, AC-T, AT and TCBP demonstrated a significant difference $(\mathrm{P}=0.022)$. In the group of patients with luminal A breast cancer, the result of the Fisher's exact test approached significant $(\mathrm{P}=0.059)$.

Conclusions: Neither PR/CR nor pCR can translate into long-term outcome benefit. PR/CR and PCR are not independent predictors in patients with advanced breast cancer. Patients who received a taxane + anthracycline regimen exhibited a higher recurrence rate than any other regimens, especially those patients with luminal A breast cancer.

Keywords: Response; neoadjuvant chemotherapy; breast cancer; outcome

Submitted Feb 09, 2020. Accepted for publication Jul 22, 2020.

doi: 10.21037/gs-20-209

View this article at: http://dx.doi.org/10.21037/gs-20-209 


\section{Introduction}

Breast cancer is the most common female malignant tumor worldwide $(1,2)$. Although a combination of topical and systemic treatments provides better prognosis than previously (3), patients with advanced breast cancer patients even if it is locally advanced breast cancer (4) continue to have poor outcomes. The development of novel methods for predicting responses and outcomes is necessary.

Neoadjuvant chemotherapy is a conventional treatment for locally advanced breast cancer $(5,6)$. This kind of treatment plays a significant role in reducing staging and breast-conserving and even axillary-conserving treatment (7-10). Whether the clinical response (CR) or pathological response (PR) to neoadjuvant chemotherapy can translate into prognostic benefit remains controversial. Romero (11) and his colleagues reported that the pathological assessment (pCR) or clinical assessment (PR/CR) of tumor response might signify the prognosis in locally advanced breast cancer patients. Asaoka (12) carried out a retrospective analysis of 1,599 patients with breast cancer who were treated with neoadjuvant chemotherapy. The results showed that patients achieving pCR to neoadjuvant chemotherapy had excellent prognosis. Cancer recurrence was predicted by high clinical staging, large tumor size, lymph node metastasis and human epidermal growth factor receptor-2-positive (HER2+) status at baseline. Similar results were observed by LeVasseur et al. in their retrospective analysis of 267 patients who had received neoadjuvant chemotherapy (13). Their research showed that five-year relapse-free survival and breast cancer-specific survival were higher in the pCR group compared to the non-pCR group. However, in the subtypes analysis, they reported that patients with triple negative breast cancer who achieved pCR improved breast cancerspecific survival and relapse-free survival significantly, but a non-significant trend was seen in the patients with human epidermal growth factor receptor-2-positive (HER-2+) and estrogen receptor-positive (ER+) subtypes. Chen (14) and his colleagues retrospectively analyzed the outcomes of 569 patients with locally advanced breast cancer who had received neoadjuvant chemotherapy before surgery. They showed that the value of clinical and pathological responses across different breast cancer subtypes: is correlated to survival in patients with ER+/progesterone receptor (PR)+ breast cancer rather than ER/PR- locally advanced breast cancer. However, different results were reported by Glück et al. (15), who used the BluePrint and MammaPrint systems to divide patients into different recurrence risks groups across different subtypes. The researchers highlighted the finding that patients with luminal A breast cancer who had been designated as low risk with MammaPrint, had a good prognosis and did not seem to benefit from chemotherapy, but a marked benefit in response survival to neoadjuvant chemotherapy was observed in patients with HER-2+ and triple negative breast cancer.

Three well-known meta-analyses were carried out to address the controversy of whether CR/PR or pCR to neoadjuvant chemotherapy results in better outcomes. The CTNeoBC pooled analysis of 12 identified international trials and 11955 patients was carried out by the US Food and Drug Administration and was published in The Lancet by Cortazar et al. (16). The results highlighted that tumor eradication from the breast and lymph nodes was associated with improved event-free survival (EFS) and overall survival (OS) than tumour eradication from the breast alone. In further analyses of the ypT0/is ypN0 group, the researchers found the association between pCR and outcomes were the strongest in patients with triple negative breast cancer and patients with HER-2+ and ER- breast cancer who had received trastuzumab treatment. However, two other metaanalyses showed a different conclusion with the CTNeoBC analysis. Korn (17) demonstrated that pCR could not be a trial-level surrogate for EFS or OS, nor is there evidence that pCR could be used reliably to screen out nonpromising agents from further drug development. A similar conclusion was obtained by Berruti et al. (18) who analyzed 29 heterogeneous neoadjuvant trials that included 14,641 patients. The results of this meta-analysis did not support the use of pCR as a surrogate end point for DFS and OS in patients with breast cancer. However, pCR may potentially meet the criterion of surrogacy with specific systemic therapies.

Based on the current research, we carried out this retrospective analysis to determine whether the PR/ $\mathrm{CR}$ and pCR to neoadjuvant chemotherapy can translate into prognostic benefits and also research whether the administration of different chemotherapy regimens influences outcomes. We present the following article in accordance with the STROBE reporting checklist (available at http://dx.doi.org/10.21037/gs-20-209).

\section{Methods}

\section{Patients and ethics statement}

A total 300 Chinese women who was diagnosed with 
invasive breast cancer through core needle biopsy and the node status was assessed through fine needle aspiration before neoadjuvant chemotherapy were initially enrolled into this study. Among these, 67 (22.3\%) patients were treated before or have been treated elsewhere, $52(17.3 \%)$ patients' treatment information were incomplete, 19 $(6.3 \%)$ patients' contact information they registered was unavailable, 17 (5.7\%) patients rejected follow-up, 10 (3.3\%) did not underwent regular follow-up were excluded. The rest 135 (45\%) patients were included in the present study.

A retrospective analysis was carried including 135 patients diagnosed with locally advanced breast cancer, who were included in a prospective database of the Third Affiliated Hospital of Kunming Medical University, Yunnan Cancer Hospital from January 2012 to December 2015. The study was conducted in accordance with the Declaration of Helsinki (as revised in 2013). The study was approved by the Ethics Committees of the Third Affiliated Hospital of Kunming Medical University, Yunnan Cancer Hospital (No.: QT 202003) and informed consent was taken from all the patients.

\section{Neoadjuvant chemotherapy treatment}

The neoadjuvant chemotherapy strategies were classified into six subgroups as follows:

(I) Thirty patients received 4-6 cycles of an anthracycline + taxane + cyclophosphamide (TAC) regimen administered intravenously (IV) every three weeks as docetaxel $75 \mathrm{mg} / \mathrm{m}^{2}$ on day 1 , doxorubicin $50 \mathrm{mg} / \mathrm{m}^{2}$ on day 1 , and cyclophosphamide $500 \mathrm{mg} / \mathrm{m}^{2}$ on day 1 .

(II) Fifteen patients received 2-5 cycles of a 5-fluorouracil + anthracycline + cyclophosphamide (FAC) regimen administered $\mathrm{IV}$ every three weeks as 5 -fluorouracil $600 \mathrm{mg} / \mathrm{m}^{2}$ on day 1 , doxorubicin $50 \mathrm{mg} / \mathrm{m}^{2}$ on day 1 , and cyclophosphamide $500 \mathrm{mg} / \mathrm{m}^{2}$ on day 1 .

(III) Twenty-five patients were treated with an anthracycline + cyclophosphamide sequential taxane (ACT) regimen administered IV as four cycles of doxorubicin $50 \mathrm{mg} / \mathrm{m}^{2}$ on day 1 and cyclophosphamide $500 \mathrm{mg} / \mathrm{m}^{2}$ on day 1 every two or three weeks followed by four cycles of docetaxel $75 \mathrm{mg} / \mathrm{m}^{2}$ on day 1 every two or three weeks.

(IV) Thirty-eight patients received four cycles of an anthracycline + taxane (AT) regimen every three weeks, administered IV as docetaxel $75 \mathrm{mg} / \mathrm{m}^{2}$ on day 1 and doxorubicin $50 \mathrm{mg} / \mathrm{m}^{2}$ on day 1 .

(V) Eleven patients received four cycles of a carboplatin + taxane (TCBP) regimen every three weeks, administered IV as docetaxel $75 \mathrm{mg} / \mathrm{m}^{2}$ on day 1 and carboplatin AUC 6 on day 1.

(VI) Sixteen patients received another neoadjuvant chemotherapy regimen.

\section{Clinical and pathological response}

Clinical and pathological responses to neoadjuvant chemotherapy were assessed based on the clinical and pathological data. The clinical response to neoadjuvant chemotherapy was evaluated by MRI and ultrasound examinations and in accordance with the response evaluation criteria in solid tumors RECIST 1.1 version $(19,20)$. The pCR after neoadjuvant chemotherapy was defined as eradication of carcinoma from both the breast and lymph nodes.

\section{Follow-up}

After systemic treatment of surgery, chemotherapy, and radiotherapy, all of the patients underwent regular followup including a tumor marker test, ultrasonography examination, and chest X-ray at every 2-3 months in the first year after surgery and every 6 months during the following 5 years and then every 12 months thereafter. Radiographs with a molybdenum target tube, breast MRI an investigation, the isotope bone scan and curettage, and general CT scans were carried out once a year (21). The results and events of all of the patients were recorded in the database.

\section{Statistical analysis}

The comparisons of clinical and pathological characteristics and recurrence were made using the chi-squared test. Fisher's exact test was used when the cell expectation was less than 6. The Student's $t$-test was used to analyze the differences between the variables reported as continuous data. Kaplan-Meier analysis was used for survival analysis, and group results were compared using the log rank test. Univariate and multivariate analyses were performed using the Cox regression analysis. All of the statistical analyses were performed with SPSS22.0 (Chicago, IL, USA) and Graphpad Prism 6.0. A P value of less than 0.05 was considered to be statistically significant. 


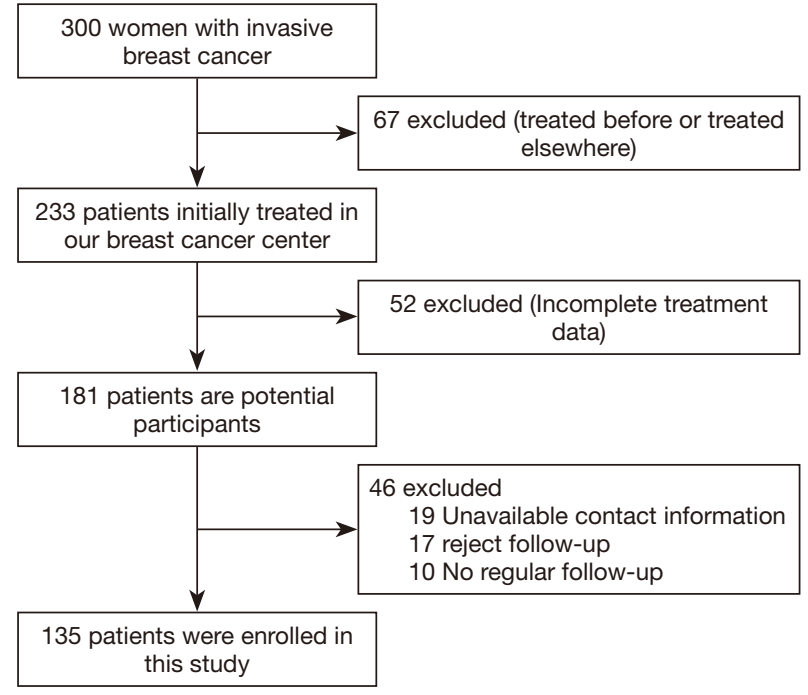

Figure 1 The flow diagram of case screen.

\section{Results}

\section{Patient and cancer characteristics}

The including of participants was exhibited in Figure 1. We first divided the patients into the PR + CR group and stable disease $(\mathrm{SD})+$ progressive disease $(\mathrm{PD})$ group according to the clinical response data. The clinical and pathological characteristics of the 135 patients with locally advanced breast cancer are summarized in Table 1 . We analyzed the correlation across two clinical response arms at the level of age, tumor size, lymph nodes status, menstruation status, chemotherapy times, Ki67 value, and molecular subtypes. The results indicated that clinical response had a tight correlation with lymph nodes status $(\mathrm{P}=0.032)$. There were no significant differences in the response between age $(\mathrm{P}=0.086)$, tumor size $(\mathrm{P}=0.398)$, menstruation status $(\mathrm{P}=0.631)$, chemotherapy times $(\mathrm{P}=0.261)$, Ki67 value $(\mathrm{P}=0.992)$ and molecular subtypes $(\mathrm{P}=0.455)$.

In the further subtype analysis, we compared the clinical response in every subtype, as summarized in the Tables S1-S4. We found that the clinical response correlated with the lymph nodes status in the luminal $\mathrm{B}$ arms $(\mathrm{P}=0.045$, Table S2). There were no significant differences in other characteristics across the subtypes There was no significant difference in other subtypes across the characteristics.
Outcomes in the different clinical and pathological response groups

After comparing the clinical and pathological characteristics, we explored whether the PR/CR and pCR to neoadjuvant chemotherapy could translate into long-term prognosis benefits. We carried out a KaplanMeier test for the 5-year OS analysis. The 5-year OS comparison of the pathological response between the pCR and non-pCR groups exhibited no significant differences $(\mathrm{P}=0.400$, Figure $2 A)$. A similar result was observed in the comparison of the clinical response between the PR/CR and SD/PD groups $(\mathrm{P}=0.108$, Figure $2 B)$. Then we used the Cox regression analysis to detect whether $\mathrm{CR}$ and $\mathrm{PR}$ are the independent factors influencing the prognosis. The results from the univariate and multivariate analyses did not support CR and PR as predictors of prognosis (Table 2). Furthermore, we researched the cancer recurrence in the patients who had shown clinical and pathological responses. In the comparison of the clinical response group there was no significant difference in cancer recurrence $(\mathrm{P}=0.496$, Table 3), nor was there a significant result in the comparison of pathological response $(\mathrm{P}=0.460$, Table 4). The recurrence sites of the pCR and non-pCR groups are shown in the pie chart (Figure 2C,D), and recurrence sites of different subtypes are shown in Figure 2E,F, G,H.

\section{Long-term outcomes between different neoadjuvant chemotherapy regimens}

We wanted to detect whether different neoadjuvant chemotherapy regimens exhibited different survival rates using the Kaplan-Meier analysis for 5-year OS. The results demonstrated there were no significant differences in survival between different regimens $(\mathrm{P}=0.307$, Figure $3 A$ ). The recurrence sites of different regimens are shown in Figure 3B,C,D,E,F,G. We focused on those patients with clinical response $\mathrm{PR} / \mathrm{CR}$ and $\mathrm{PD} / \mathrm{SD}$ who received different neoadjuvant chemotherapy regimens. In the PR/ $\mathrm{CR}$ and $\mathrm{PD} / \mathrm{SD}$ comparison of every single regimen, the result showed no significant differences in survival (Table 5). However, for those PR/CR patients, results demonstrated a significant difference in a comparison of the regimens with P 0.022 (Table 6). 
Table 1 Comparison of clinicopathological characteristics between CR + PR group and SD + PD group

\begin{tabular}{|c|c|c|c|c|c|c|c|}
\hline Variables & $\begin{array}{c}\text { Total } \\
(n=135)\end{array}$ & $\begin{array}{c}C R+P R \\
(n=97)\end{array}$ & $\begin{array}{c}S D+P D \\
(n=38)\end{array}$ & $\begin{array}{l}\text { Chi-square } \\
\text { value }\end{array}$ & $P$ value & $\begin{array}{l}\text { Hazard } \\
\text { ratio }\end{array}$ & $95 \% \mathrm{Cl}$ \\
\hline Age (years) & & & & 0.020 & 0.886 & 1.058 & $0.490-2.281$ \\
\hline$\geq 50$ & 52 & 37 & 15 & & & & \\
\hline T3 & 11 & 10 & 1 & & & & \\
\hline $\mathrm{T} 4$ & 13 & 9 & 4 & & & & \\
\hline Lymph nodes status & & & & 4.581 & 0.032 & 0.429 & $0.196-0.940$ \\
\hline Postmenopausal & 49 & 34 & 15 & & & & \\
\hline Premenopausal & 86 & 63 & 23 & & & & \\
\hline Chemotherapy times & & & & 1.266 & 0.261 & 1.824 & $0.633-5.250$ \\
\hline$\leq 4$ & 26 & 21 & 5 & & & & \\
\hline $\begin{array}{l}>51097633 \leq 426215>51097633 \leq 426215> \\
51097633 \leq 426215>5\end{array}$ & 109 & 76 & 33 & & & & \\
\hline Ki67 value & & & & 0.000 & 0.992 & 1.004 & $0.437-2.307$ \\
\hline HER-2 + & 16 & 14 & 2 & & & & \\
\hline TNBC & 25 & 17 & 8 & & & & \\
\hline
\end{tabular}

NS, no significance; CR, complete response; PR, partial response; SD, stable disease; PD, progressive disease.

\section{Different treatment regimens in different subtypes}

Based on the findings above, we further examined how different neoadjuvant chemotherapy regimens influenced cancer recurrence in different subtypes. We focused on comparing the five main regimens: TAC, FAC, AC-T, AT and TCBP regimens. In the group of patients with luminal A group breast cancer, results from the Fisher's exact test approached significance $(\mathrm{P}=0.059$, Table 7$)$. There were no significant differences in cancer recurrence among patients with luminal B breast cancer $(\mathrm{P}=0.715$, Table 8$)$, those with
HER-2+ tumors $(\mathrm{P}>0.999$, Table 9), or those who were treated with TNBC $(\mathrm{P}>0.999$, Table 10).

\section{Discussion}

The correlation between the pCR to neoadjuvant chemotherapy and outcomes was first reported in the landmark National Surgical Adjuvant Breast and Bowel Project B-18 and B-27 trials $(22,23)$. The FDA and the European Medicines Agency declared that, after accelerated 


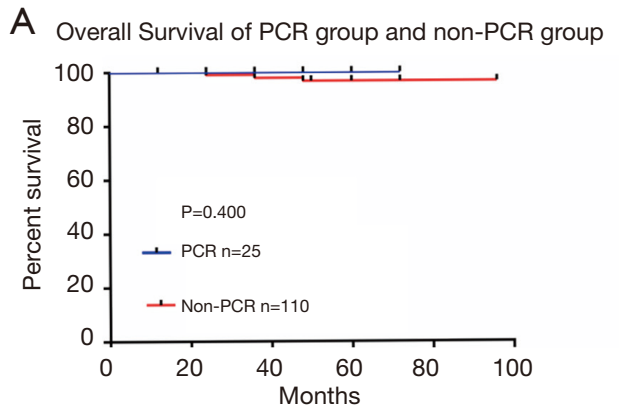

C

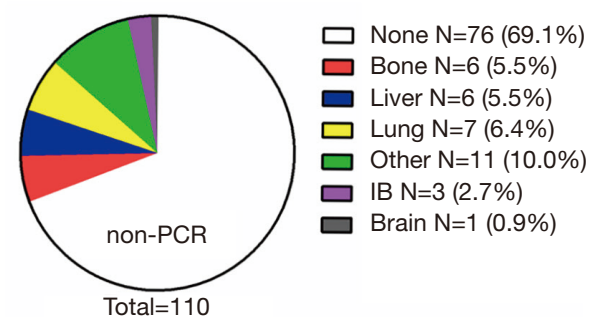

E

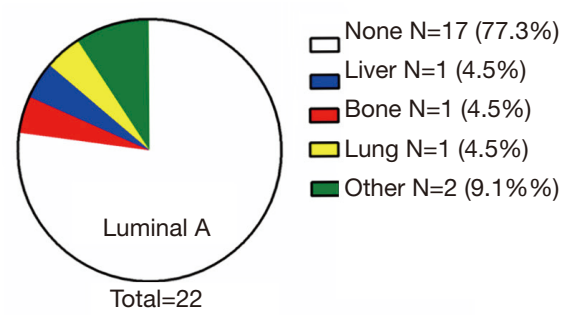

G

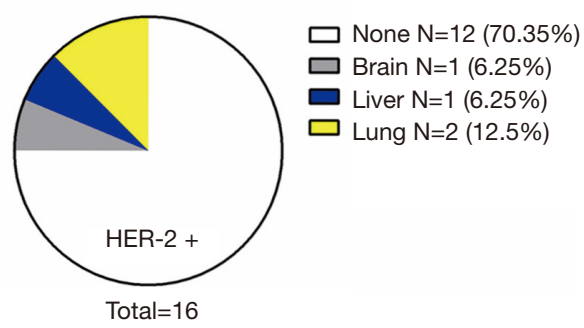

B Overall Survival of clinical PR/CP group and SD/PD group

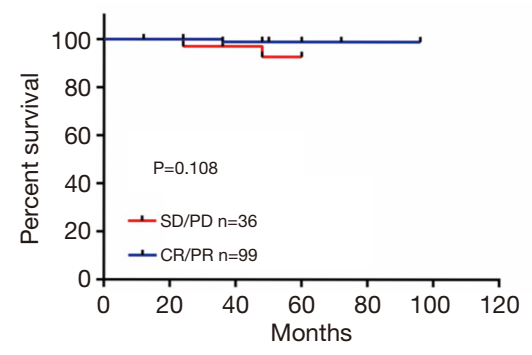

D

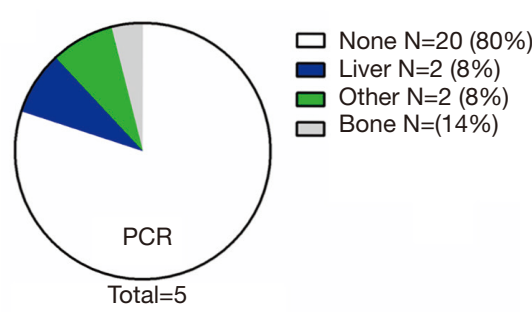

$\mathrm{F}$

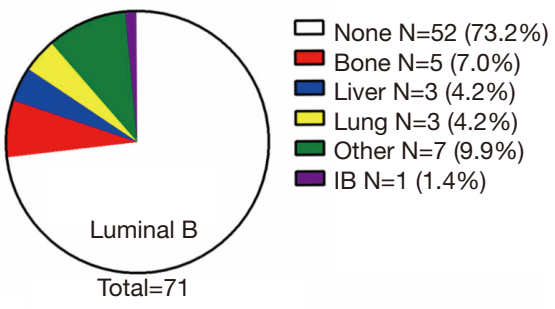

$\mathrm{H}$

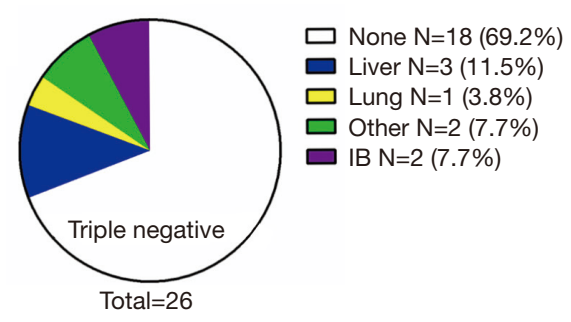

Figure 2 Overall Survival of pathology and clinical response. (A) OS analyzed by Kaplan-Meier curves for breast cancer patients with pathology PCR group ( $n=25)$ versus non-PCR group ( $n=110)$. (B) OS of patients with PR/CR group ( $n=99)$ versus SD/PD group (n=36). (C,D) Pie charts of PCR group and non-PCR group recurrent sites. (E) Recurrence pie chart of luminal A patients (n=22). (F) Recurrence pie chart of luminal B patients ( $n=71)$. (G) Recurrence pie chart of HER-2 + patients ( $n=16)$. (H) Recurrence pie chart of triple negative patients $(\mathrm{n}=26)$.

approval, demonstration of an improvement in OS and disease-free survival after neoadjuvant chemotherapy would be required $(24,25)$.

In this study, we analyzed the clinical and pathological characteristics of patients and the response to neoadjuvant chemotherapy and found that the lymph nodes status correlates with response to therapy meaning patients with lymph node metastasis may obtain a better response to neoadjuvant chemotherapy. The result is similar to the finding of Caudle (26) and Tee (27). This phenomenon was 
Table 2 Cox regression analysis for overall survival

\begin{tabular}{|c|c|c|c|c|c|c|}
\hline Variables & \multicolumn{3}{|c|}{ Univariate analysis } & \multicolumn{3}{|c|}{ Multivariate analysis } \\
\hline Age & 0.991 & $0.856-1.148$ & 0.906 & 0.980 & $0.732-1.312$ & 0.890 \\
\hline Tumor invasion depth & 1.790 & $0.488-6.567$ & 0.380 & 1.861 & $0.460-7.531$ & 0.384 \\
\hline Lymph node metastasis & 35.099 & NS & 0.504 & 0.861 & $0.595-1.244$ & 0.425 \\
\hline KI67 expression & 35.176 & NS & 0.503 & NS & NS & 0.983 \\
\hline Chemotherapy times & 0.456 & $0.041-5.034$ & 0.618 & 0.139 & $0.04-4.849$ & 0.276 \\
\hline Pathology response to NCT (PCR) & 27.818 & NS & 0.600 & 1.389 & $0.894-2.156$ & 0.144 \\
\hline Clinical response to NCT (PR/NonPR) & 35.478 & $0.516-62.986$ & 0.156 & 12.252 & $0.639-274.661$ & 0.095 \\
\hline
\end{tabular}

NS, no significance; PCR, pathologic complete response; PR, partial response.

Table 3 The recurrence between clinical PR/CP group and SD/PD group

\begin{tabular}{lccccccc}
\hline Events & Total $(\mathrm{n}=135)$ & $\mathrm{PR} / \mathrm{CR}(\mathrm{n}=98)$ & SD/PD $(\mathrm{n}=37)$ & Chi-square value & $\mathrm{P}$ value & Hazard ratio & 95\% Cl \\
\hline Recurrence & 38 & 26 & 12 & 0.463 & 0.496 & 0.752 & $0.331-1.711$ \\
Non-recurrence & 97 & 72 & 25 & & & \\
\hline
\end{tabular}

$\mathrm{CR}$, complete response; PR, partial response; SD, stable disease; $\mathrm{PD}$, progressive disease; $\mathrm{Cl}$, confidence interval.

Table 4 The recurrence between pathology PCR group and non-PCR group

\begin{tabular}{lccccccr}
\hline Events & Total $(n=135)$ & PCR $(n=24)$ & non-PCR $(n=111)$ & Chi-square value & $P$ value & Hazard ratio & $95 \%$ Cl \\
\hline Recurrence & 38 & 5 & 33 & 0.772 & 0.460 & 0.622 & $0.214-1.806$ \\
Non-recurrence & 97 & 19 & 78 & & & \\
\hline
\end{tabular}

$\mathrm{Cl}$, confidence interval; PCR, pathologic complete response.

highlighted in patients with luminal B breast cancer. Breast cancer with luminal B may potentially contribute to axillary lymph node conservation and sentinel lymph node biopsy after neoadjuvant chemotherapy.

In the present study, we researched the key scientific problem of whether PR/CR and pCR to neoadjuvant chemotherapy can transfer into long-term prognosis benefits. Our research demonstrated that neither clinical response PR/CR nor pCR translated into long-term prognosis benefit. Univariate and multivariate analyses performed with the Cox regression analysis also showed that $\mathrm{PR} / \mathrm{CR}$ and $\mathrm{pCR}$ are not the factors influencing prognosis. Our research findings differ from the findings of Romero (11), Asaoka (12), and Cortazar (16). However, our results were similar to those reported by Korn (17) and Berruti (18). It is possible that most of the clinical trials data or retrospective analyses have the sample patients from homogeneous populations such that a meaningful correlation of $\mathrm{pCR}$ or PR/CR between EFS or OS trial results might not have been seen. Our results also differ from those of Chen (14), although both our research and Chen's included Chinese patients. Most patients from our sample lived in Yunnan Province, an area with many ethnic minorities. This sample may have provided different outcomes than would have been observed if we had sampled Han people, who represent the largest population in China. We also noticed that some of our patients showed a clinical response PR and SD. Most of these patients had undergone surgery treatment before completion of their entire course of neoadjuvant chemotherapy and after the surgery, most of them received a different chemotherapy regimen. Switching to a different regimen may have been an important factor 
A

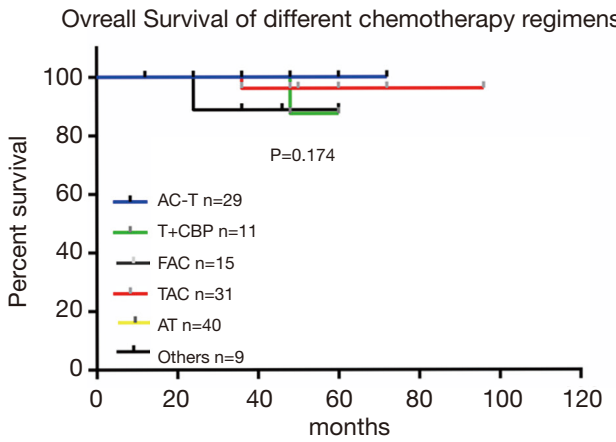

Note. TAC Anthracycline + cyclophosphamide + taxane T + CBP Taxane + carboplatin FAC 5-fluorouracil + anthracycline + cyclophosphamide AT Taxane + anthracycline AC-T Anthracycline + cyclophosphamide sequential taxane

B

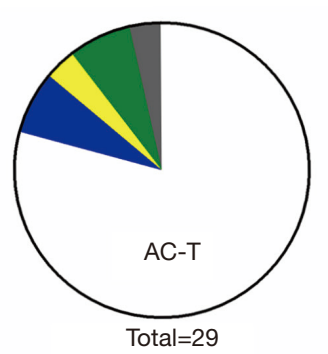

$\mathrm{D}$

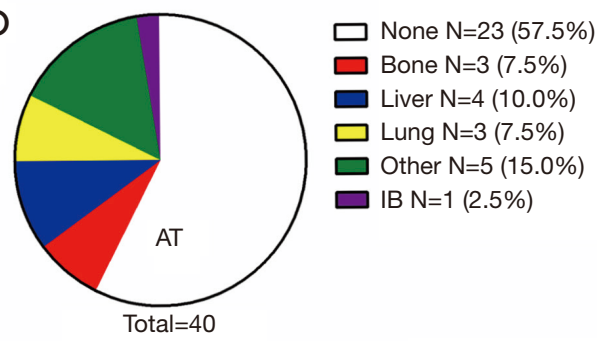

$\mathrm{F}$

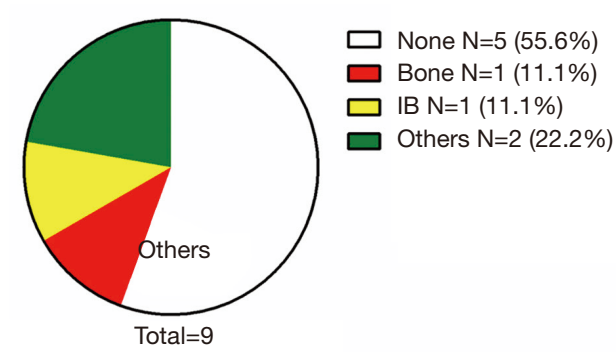

C

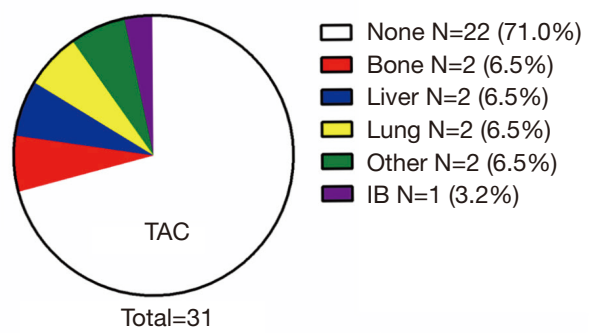

$\mathrm{E}$

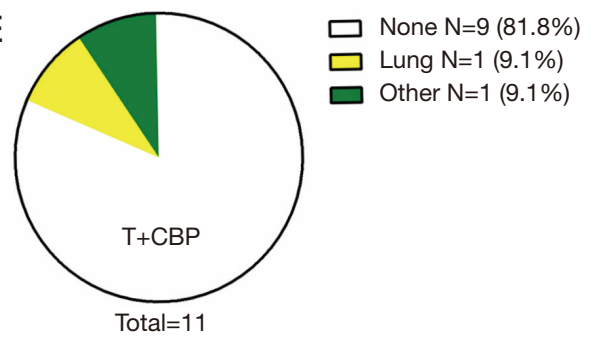

G

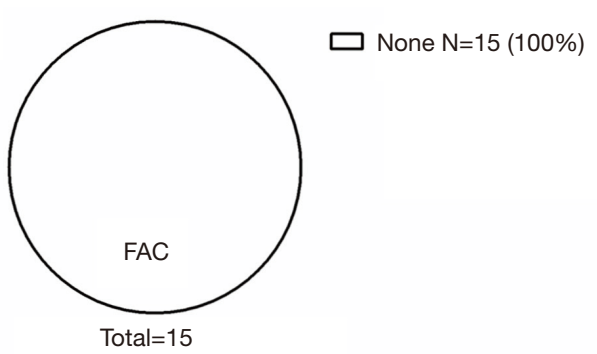

Figure 3 (A) OS analyzed by Kaplan-Meier curves for breast cancer patients with different chemotherapy regimens (anthracycline + cyclophosphamide sequential taxane $\mathrm{n}=29$, anthracycline + cyclophosphamide + taxane $\mathrm{n}=31$, taxane + carboplatin $\mathrm{n}=11,5$-fluorouracil + anthracycline + cyclophosphamide $n=15$, taxane + anthracycline $n=40$, others $n=9$ ). (B) Pie charts of recurrent site in patients with anthracycline + cyclophosphamide sequential taxane regimen. (C) Pie charts of recurrent site in patients with anthracycline + cyclophosphamide + taxane regimen. (D) Pie charts of recurrent site in patients with taxane + anthracycline regimen. (E) Pie charts of recurrent site in patients with taxane + carboplatin regimen. (F) Pie charts of recurrent site in patients with others regimens. (G) Pie charts of recurrent site in patients with 5 -fluorouracil + anthracycline + cyclophosphamide regimen. 
Table 5 Recurrence of PR/CR group comparison SD/PD group between different chemotherapy regimens

\begin{tabular}{|c|c|c|c|c|c|c|c|}
\hline Regimens and clinical response & $\begin{array}{c}\text { Total } \\
(n=135)\end{array}$ & $\begin{array}{l}\text { Recurrence } \\
\quad(n=41)\end{array}$ & $\begin{array}{l}\text { Non-recurrence } \\
(n=94)\end{array}$ & $\begin{array}{l}\text { Chi-square } \\
\text { value }\end{array}$ & $P$ value & $\begin{array}{l}\text { Hazard } \\
\text { ratio }\end{array}$ & $95 \% \mathrm{Cl}$ \\
\hline $\begin{array}{l}\text { Anthracycline + cyclophosphamide } \\
\text { sequential taxane }(n=29)\end{array}$ & & & & 0.075 & $>0.999$ & 1.389 & $0.131-14.779$ \\
\hline $\mathrm{PR} / \mathrm{CR}$ & 23 & 5 & 18 & & & & \\
\hline PD/SD & 6 & 1 & 5 & & & & \\
\hline $\mathrm{PR} / \mathrm{CR}$ & 25 & 7 & 18 & & & & \\
\hline $\mathrm{PD} / \mathrm{SD}$ & 6 & 2 & 4 & & & & \\
\hline Taxane + carboplatin $(n=11)$ & & & & 4.278 & 0.109 & NS & NS \\
\hline $\begin{array}{l}\text { 5-fluorouracil + anthracycline + } \\
\text { cyclophosphamide }(n=15)\end{array}$ & & & & NS & NS & NS & NS \\
\hline $\mathrm{PR} / \mathrm{CR}$ & 9 & 0 & 9 & & & & \\
\hline $\mathrm{PD} / \mathrm{SD}$ & 6 & 0 & 6 & & & & \\
\hline Taxane + anthracycline $(n=40)$ & & & & 0.395 & 0.530 & 0.647 & $0.166-2.527$ \\
\hline $\mathrm{PR} / \mathrm{CR}$ & 28 & 11 & 17 & & & & \\
\hline $\mathrm{PD} / \mathrm{SD}$ & 12 & 6 & 6 & & & & \\
\hline Others $(n=9)$ & & & & 0.032 & $>0.999$ & 0.750 & $0.032-17.507$ \\
\hline
\end{tabular}

NS, no significance; CR, complete response; PR, partial response; SD, stable disease; PD, progressive disease; $\mathrm{Cl}$, confidence interval.

Table 6 Recurrence of PR/CR group between different chemotherapy regimens

\begin{tabular}{|c|c|c|c|c|c|c|c|}
\hline Regimens and clinical response & $\begin{array}{c}\text { Total } \\
(\mathrm{n}=96)\end{array}$ & $\begin{array}{l}\text { Recurrence } \\
\qquad(\mathrm{n}=26)\end{array}$ & $\begin{array}{l}\text { Non-recurrence } \\
\qquad(n=70)\end{array}$ & $\begin{array}{l}\text { Chi-square } \\
\text { value }\end{array}$ & $P$ value & $\begin{array}{l}\text { Hazard } \\
\text { ratio }\end{array}$ & $\begin{array}{c}95 \% \\
\mathrm{Cl}\end{array}$ \\
\hline Anthracycline + cyclophosphamide sequential taxane & 23 & 5 & 18 & 13.048 & 0.022 & NS & NS \\
\hline Anthracycline + cyclophosphamide + taxane & 25 & 7 & 18 & & & & \\
\hline Taxane + carboplatin & 7 & 0 & 7 & & & & \\
\hline Taxane + anthracycline & 28 & 11 & 17 & & & & \\
\hline Others & 4 & 3 & 1 & & & & \\
\hline
\end{tabular}

NS, no significance; $\mathrm{Cl}$, confidence interval; CR, complete response; PR, partial response. 
Table 7 Recurrence of PR/CR group between different chemotherapy regimens of luminal A patients

\begin{tabular}{|c|c|c|c|c|c|c|c|}
\hline Regimens and clinical response & $\begin{array}{l}\text { Total } \\
(n=13)\end{array}$ & $\begin{array}{l}\text { Recurrence } \\
\qquad(n=4)\end{array}$ & $\begin{array}{l}\text { Non-recurrence } \\
\qquad(n=9)\end{array}$ & $\begin{array}{l}\text { Chi-square } \\
\text { value }\end{array}$ & $P$ value & $\begin{array}{l}\text { Hazard } \\
\text { ratio }\end{array}$ & $\begin{array}{c}95 \% \\
\mathrm{Cl}\end{array}$ \\
\hline Anthracycline + cyclophosphamide sequential taxane & 5 & 1 & 4 & 9.244 & 0.059 & NS & NS \\
\hline Anthracycline + cyclophosphamide + taxane & 2 & 0 & 2 & & & & \\
\hline Taxane + carboplatin & 1 & 0 & 1 & & & & \\
\hline Taxane + anthracycline & 3 & 3 & 0 & & & & \\
\hline
\end{tabular}

NS, no significance; $\mathrm{Cl}$, confidence interval; $\mathrm{CR}$, complete response; $\mathrm{PR}$, partial response.

Table 8 Recurrence of PR/CR group between different chemotherapy regimens of luminal B patients

\begin{tabular}{|c|c|c|c|c|c|c|c|}
\hline Regimens and clinical response & $\begin{array}{l}\text { Total } \\
(n=46)\end{array}$ & $\begin{array}{l}\text { Recurrence } \\
\quad(n=12)\end{array}$ & $\begin{array}{l}\text { Non-recurrence } \\
\qquad(n=34)\end{array}$ & $\begin{array}{c}\text { Chi-square } \\
\text { value }\end{array}$ & $P$ value & $\begin{array}{c}\text { Hazard } \\
\text { ratio }\end{array}$ & $\begin{array}{c}95 \% \\
\mathrm{Cl}\end{array}$ \\
\hline Anthracycline + cyclophosphamide sequential taxane & 12 & 2 & 10 & 2.397 & 0.715 & NS & NS \\
\hline Taxane + carboplatin & 1 & 0 & 1 & & & & \\
\hline
\end{tabular}

NS, no significance; $\mathrm{Cl}$, confidence interval; $\mathrm{CR}$, complete response; PR, partial response.

Table 9 Recurrence of PR/CR group between different chemotherapy regimens of HER-2 positive patients

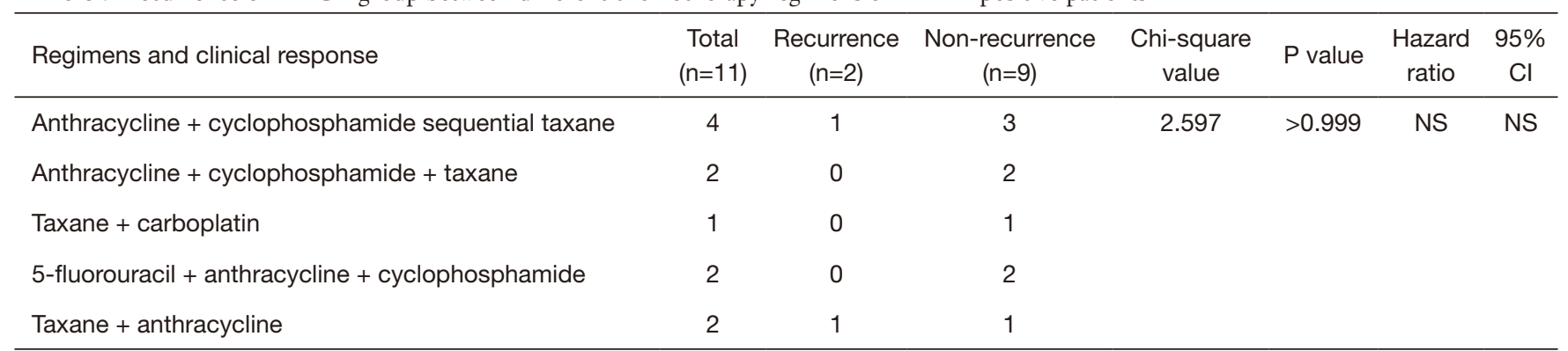

NS, no significance; $\mathrm{Cl}$, confidence interval; CR, complete response; PR, partial response.

Table 10 Recurrence of PR/CR group between different chemotherapy regimens of TNBC patients

\begin{tabular}{|c|c|c|c|c|c|c|c|}
\hline Regimens and clinical response & $\begin{array}{c}\text { Total } \\
(n=17)\end{array}$ & $\begin{array}{l}\text { Recurrence } \\
\qquad(n=3)\end{array}$ & $\begin{array}{l}\text { Non-recurrence } \\
\qquad(n=14)\end{array}$ & $\begin{array}{l}\text { Chi-square } \\
\text { value }\end{array}$ & $P$ value & $\begin{array}{l}\text { Hazard } \\
\text { ratio }\end{array}$ & $\begin{array}{c}95 \% \\
\mathrm{Cl}\end{array}$ \\
\hline Anthracycline + cyclophosphamide sequential taxane & 0 & 0 & 0 & & & & \\
\hline Anthracycline + cyclophosphamide + taxane & 7 & 2 & 5 & 1.436 & $>0.999$ & NS & NS \\
\hline Taxane + carboplatin & 2 & 0 & 2 & & & & \\
\hline Taxane + anthracycline & 6 & 1 & 5 & & & & \\
\hline
\end{tabular}

NS, no significance; Cl, confidence interval; CR, complete response; PR, partial response, TNBC, triple negative breast cancer. 
influencing the long-term outcomes.

Besides the correlation between long-term outcomes and clinical response or pathological response to neoadjuvant chemotherapy, many scholars have focused on the relationship of other aspects of neoadjuvant chemotherapy with outcomes. Alba (28) reported that a Ki67 proliferation index greater than $50 \%$ may be an independent predictor for pCR to neoadjuvant chemotherapy, and they declared that cell proliferation may be tightly correlated with chemosensitivity. Baulies et al. (29) illustrated timedependent prognostic factors. Distant recurrencefree intervals in patients with breast cancer who receive neoadjuvant chemotherapy are influenced by achieving pCR and the cancer subtype. Patients with more aggressive biological behaviour have poorer outcomes during the first 5 years and patients with $\mathrm{HR}+$ breast cancer remain at risk for distant recurrence for many years. These scholars focused more on the biological behavior of breast cancer and outcomes rather than the correlation of chemotherapy regimens and prognoses. Schettini (30) and Li (31) performed meta-analysis and reported that most research focused on the chemotherapy regimens on HER-2+ and triple negative breast cancer. There are no reports comparing multiple types of regimens across clinical response and pathological response or comparing these outcomes across all kinds of subtypes. This is the first study to research this aspect of breast cancer treatment, and we found no significant differences in outcomes in the comparison of regimens, including TAC, AC-T, FAC, TCBP, AT and others. Moreover, in the PR/CR and PD/ $\mathrm{SD}$ comparison of every single regimen, the result showed no significant differences. However, for the PR/CR patients, results from the comparison of regimens showed a significant difference. We believe the use of all of the regimens presented here is feasible for clinicians who use the PR/CR response to guide treatment of patients with breast cancer. However, we noticed that 40 patients who received the taxane + anthracycline regimen exhibited a higher recurrence rate than the rate in any other regimens, this concerning phenomenon was particularly observed in the recurrence comparison of regimens in the PR/CR group. Therefore, we recommend avoiding the taxane + anthracycline regimen in the neoadjuvant chemotherapy to improve outcomes. Conversely, cyclophosphamide treatment exhibited a significant effect, as patients treated with taxane and anthracycline combined with cyclophosphamide showed a lower recurrence rate than taxane and anthracycline alone. According to our findings, we recommend cyclophosphamide be added to neoadjuvant chemotherapy regimens of taxane and anthracycline.

When we compared the results across each neoadjuvant chemotherapy subtype, we detected five main regimens for comparison. In the group of patients with luminal A breast cancer, results of the Fisher's exact test approached significance. We examined the data and found that cancer in all of the patients with luminal A breast cancer who received taxane + anthracycline recurred. The phenomenon was not exhibited in other subtypes and in other regimens administered to patients with luminal A breast cancer. Thus, we strongly recommend avoiding the use of the taxane + anthracycline neoadjuvant chemotherapy regimen for patients with luminal A breast cancer. Because the clinical samples in this study were small, we hope to obtain more convincing conclusions by increasing the sample size in future research.

To sum up, in this novel study, we illustrated that neither clinical response PR/CR nor pCR can transfer into long-term outcome benefit. Clinical response PR/CR and pathological response pCR are not the independent prognostic predictors in breast cancer. We are the first to report that patients who received taxane + anthracycline regimen exhibited a higher recurrence rate than any other regimens, especially for those patients with luminal A breast cancer. We hope our research can help guide clinicians in choosing the appropriate neoadjuvant chemotherapy regimens for breast cancer.

\section{Acknowledgments}

Funding: The present study was supported by the National Natural Science Foundation of China (grant No. 81960542 and 81960517) and Scientific Research Foundation of Yunnan Education Department (grant Nos. 2019J1288 and 2020J0198).

\section{Footnote}

Reporting Checklist: The authors have completed the STROBE Reporting checklist. Available at http://dx.doi. org/10.21037/gs-20-209

Data Sharing Statement: Available at http://dx.doi. org/10.21037/gs-20-209

Conflicts of Interest: All authors have completed the ICMJE uniform disclosure form (available at http://dx.doi. 
org/10.21037/gs-20-209). The authors have no conflicts of interest to declare.

Ethical Statement: The authors are accountable for all aspects of the work in ensuring that questions related to the accuracy or integrity of any part of the work are appropriately investigated and resolved. The study was conducted in accordance with the Declaration of Helsinki (as revised in 2013). The study was approved by the Ethics Committees of the Third Affiliated Hospital of Kunming Medical University, Yunnan Cancer Hospital (No. QT 202003) and informed consent was taken from all the patients.

Open Access Statement: This is an Open Access article distributed in accordance with the Creative Commons Attribution-NonCommercial-NoDerivs 4.0 International License (CC BY-NC-ND 4.0), which permits the noncommercial replication and distribution of the article with the strict proviso that no changes or edits are made and the original work is properly cited (including links to both the formal publication through the relevant DOI and the license). See: https://creativecommons.org/licenses/by-nc-nd/4.0/.

\section{References}

1. Siegel RL, Miller KD, Jemal A. Cancer statistics, 2020. CA Cancer J Clin 2020;70:7-30.

2. DeSantis CE, Ma J, Gaudet MM, et al. Breast cancer statistics, 2019. CA Cancer J Clin 2019;69:438-51.

3. Gourd K. San Antonio Breast Cancer Symposium 2019. Lancet Oncol 2020;21:28.

4. Tryfonidis K, Senkus E, Cardoso MJ, et al. Management of locally advanced breast cancer-perspectives and future directions. Nat Rev Clin Oncol 2015;12:147-62.

5. Mathew J, Asgeirsson KS, Cheung KL, et al. Neoadjuvant chemotherapy for locally advanced breast cancer: a review of the literature and future directions. Eur J Surg Oncol 2009;35:113-22.

6. O'Halloran N, Lowery A, Curran C, et al. A Review of the Impact of Neoadjuvant Chemotherapy on Breast Surgery Practice and Outcomes. Clin Breast Cancer 2019;19:377-82.

7. Russo L, Maltese A, Betancourt L, et al. Locally advanced breast cancer: Tumor-infiltrating lymphocytes as a predictive factor of response to neoadjuvant chemotherapy. Eur J Surg Oncol 2019;45:963-8.

8. Sun Y, Liao M, He L, et al. Comparison of breast- conserving surgery with mastectomy in locally advanced breast cancer after good response to neoadjuvant chemotherapy: A PRISMA-compliant systematic review and meta-analysis. Medicine (Baltimore) 2017;96:e8367.

9. Bi Z, Liu J, Chen P, et al. Neoadjuvant chemotherapy and timing of sentinel lymph node biopsy in different molecular subtypes of breast cancer with clinically negative axilla. Breast Cancer 2019;26:373-7.

10. Vriens BEPJ, Keymeulen KBMI, Kroep JR, et al. Axillary staging in breast cancer patients treated with neoadjuvant chemotherapy in two Dutch phase III studies. Oncotarget 2017;8:46557-64.

11. Romero A, García-Sáenz JA, Fuentes-Ferrer M, et al. Correlation between response to neoadjuvant chemotherapy and survival in locally advanced breast cancer patients. Ann Oncol 2013;24:655-61.

12. Asaoka M, Narui K, Suganuma N, et al. Clinical and pathological predictors of recurrence in breast cancer patients achieving pathological complete response to neoadjuvant chemotherapy. Eur J Surg Oncol 2019;45:2289-94.

13. LeVasseur N, Sun J, Gondara L, et al. Impact of pathologic complete response on survival after neoadjuvant chemotherapy in early-stage breast cancer: a populationbased analysis. J Cancer Res Clin Oncol 2020;146:529-36.

14. Chen S, Liu Y, Ouyang QW, et al. Clinical and pathological response to neoadjuvant chemotherapy based on primary tumor reduction is correlated to survival in hormone receptor-positive but not hormone receptornegative locally advanced breast cancer. Ann Surg Oncol 2015;22:32-9.

15. Glück S, de Snoo F, Peeters J, et al. Molecular subtyping of early-stage breast cancer identifies a group of patients who do not benefit from neoadjuvant chemotherapy. Breast Cancer Res Treat 2013;139:759-67.

16. Cortazar P, Zhang L, Untch M, et al. Pathological complete response and long-term clinical benefit in breast cancer: the CTNeoBC pooled analysis. Lancet 2014;384:164-72.

17. Korn EL, Sachs MC, McShane LM. et al. Statistical controversies in clinical research: assessing pathologic complete response as a trial-level surrogate end point for early-stage breast cancer. Ann Oncol 2016;27:10-5.

18. Berruti A, Amoroso V, Gallo F, et al. Pathologic complete response as a potential surrogate for the clinical outcome in patients with breast cancer after neoadjuvant therapy: a meta-regression of 29 randomized prospective studies. J Clin Oncol 2014;32:3883-91. 
19. Watanabe H, Okada M, Kaji Y, et al. New response evaluation criteria in solid tumours-revised RECIST guideline (version 1.1). Gan To Kagaku Ryoho 2009;36:2495-501.

20. Eisenhauer EA, Therasse P, Bogaerts J, et al. New response evaluation criteria in solid tumours: revised RECIST guideline (version 1.1). Eur J Cancer 2009;45:228-47.

21. Tang S, Zhang Q, Tang X, et al. Long-term comparisons of the efficacy, safety, and pregnancy outcomes of adjuvant tamoxifen plus ovarian function suppression in premenopausal Han and Zhuang Chinese patients with hormone receptor-positive early breast cancer. J Int Med Res 2019;47:641-52.

22. Mieog JS, van der Hage JA, van de Velde CJ. Preoperative chemotherapy for women with operable breast cancer. Cochrane Database Syst Rev 2007;(2):CD005002.

23. Kong X, Moran MS, Zhang N, et al. Meta-analysis confirms achieving pathological complete response after neoadjuvant chemotherapy predicts favourable prognosis for breast cancer patients. Eur J Cancer 2011;47:2084-90.

24. Prowell TM, Pazdur R. Pathological complete response and accelerated drug approval in early breast cancer. $\mathrm{N}$ Engl J Med 2012;366:2438-41.

25. Gianni L, Pienkowski T, Im YH, et al. Efficacy and safety of neoadjuvant pertuzumab and trastuzumab in women with locally advanced, inflammatory, or early HER2-positive breast cancer (NeoSphere): a randomised multicentre, open-label, phase 2 trial. Lancet Oncol

Cite this article as: Tang S, Wang $\mathrm{K}$, Zheng $\mathrm{K}$, Liu J, Zhang H, Tan M, Li H, Li H, Tan X, Liu D, Guo R. Clinical and pathological response to neoadjuvant chemotherapy with different chemotherapy regimens predicts the outcome of locally advanced breast cancer. Gland Surg 2020;9(5):1415-1427. doi: $10.21037 / g s-20-209$
2012;13:25-32.

26. Caudle AS, Yang WT, Krishnamurthy S, et al. Improved Axillary Evaluation Following Neoadjuvant Therapy for Patients With Node-Positive Breast Cancer Using Selective Evaluation of Clipped Nodes: Implementation of Targeted Axillary Dissection. J Clin Oncol 2016;34:1072-8.

27. Tee SR, Devane LA, Evoy D, et al. Meta-analysis of sentinel lymph node biopsy after neoadjuvant chemotherapy in patients with initial biopsy-proven nodepositive breast cancer. Br J Surg 2018;105:1541-52.

28. Alba E, Lluch A, Ribelles N, et al. High Proliferation Predicts Pathological Complete Response to Neoadjuvant Chemotherapy in Early Breast Cancer. Oncologist 2016;21:778.

29. Baulies S, Belin L, Mallon P, et al. Time-varying effect and long-term survival analysis in breast cancer patients treated with neoadjuvant chemotherapy. Br J Cancer 2015;113:30-6.

30. Schettini F, Pascual T, Conte B, et al. HER2-enriched subtype and pathological complete response in HER2positive breast cancer: A systematic review and metaanalysis. Cancer Treat Rev 2020;84:101965.

31. Li Y, Yang D, Chen P, et al. Efficacy and safety of neoadjuvant chemotherapy regimens for triple-negative breast cancer: a network meta-analysis. Aging (Albany NY) 2019;11:6286-311. 


\section{Supplementary}

Table S1 Comparison of clinicopathological characteristics between CR + PR group and SD + PD group in luminal A patients

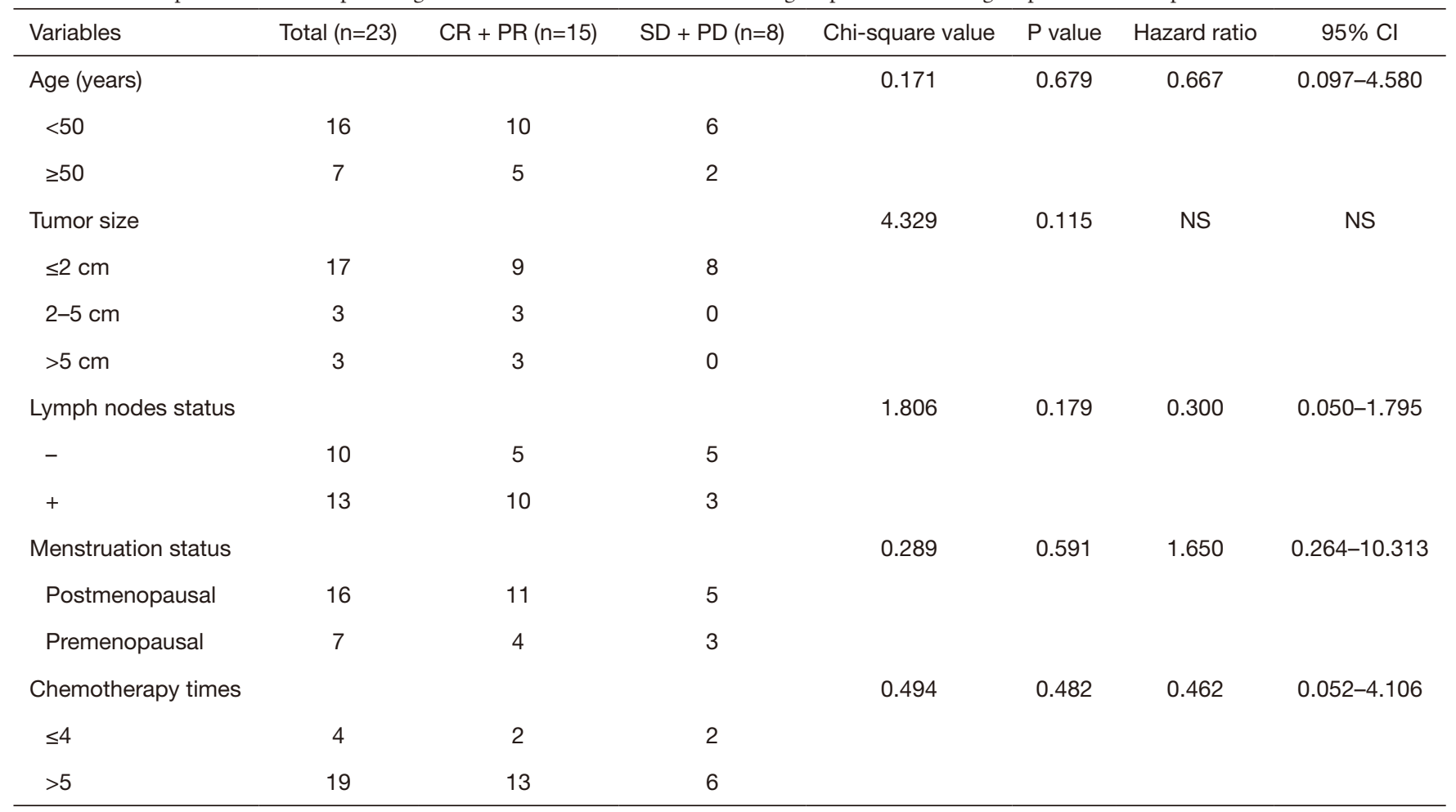

NS, no significance; CR, complete response; PR, partial response; SD, stable disease; PD, progressive disease.

Table S2 Comparison of clinicopathological characteristics between CR + PR group and SD + PD group in luminal B patients

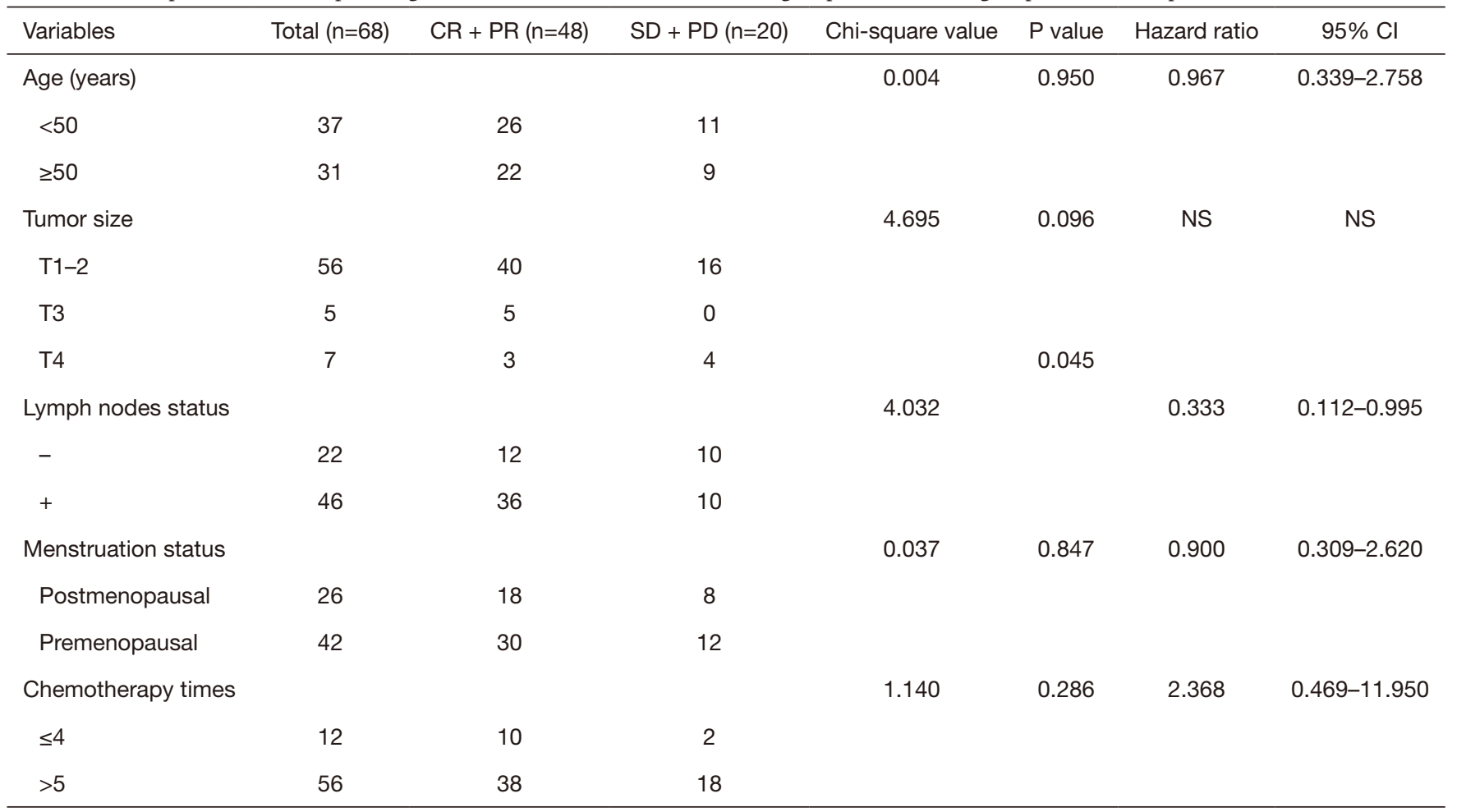

NS, no significance; CR, complete response; PR, partial response; SD, stable disease; PD, progressive disease. 
Table S3 Comparison of clinicopathological characteristics between CR + PR group and SD + PD group in HER-2+ patients

\begin{tabular}{|c|c|c|c|c|c|c|c|}
\hline Variables & Total $(n=16)$ & $C R+P R(n=14)$ & $\mathrm{SD}+\mathrm{PD}(\mathrm{n}=2)$ & Chi-square value & $P$ value & Hazard ratio & $95 \% \mathrm{Cl}$ \\
\hline$<50$ & 10 & 9 & 1 & & & & \\
\hline$\geq 50$ & 6 & 5 & 1 & & & & \\
\hline Tumor size & & & & 3.048 & 0.218 & NS & NS \\
\hline T3 & 2 & 1 & 1 & & & & \\
\hline $\mathrm{T} 4$ & 2 & 2 & 0 & & & & \\
\hline Lymph nodes status & & & & 0.152 & 0.696 & 0.000 & $0.000-0.000$ \\
\hline- & 1 & 1 & 0 & & & & \\
\hline Postmenopausal & 10 & 9 & 1 & & & & \\
\hline Premenopausal & 6 & 5 & 1 & & & & \\
\hline Chemotherapy times & & & & 0.327 & 0.568 & 0.000 & $0.469-11.950$ \\
\hline$\leq 4$ & 2 & 2 & 0 & & & & \\
\hline$>5$ & 14 & 12 & 2 & & & & \\
\hline Ki-67 value & & & & 0.762 & 0.383 & 0.273 & $0.013-5.769$ \\
\hline$<14$ & 4 & 3 & 1 & & & & \\
\hline$\geq 14$ & 12 & 11 & 1 & & & & \\
\hline
\end{tabular}

NS, no significance; CR, complete response; PR, partial response; SD, stable disease; PD, progressive disease. 
Table S4 Comparison of clinicopathological characteristics between CR + PR group and SD + PD group in TN patients

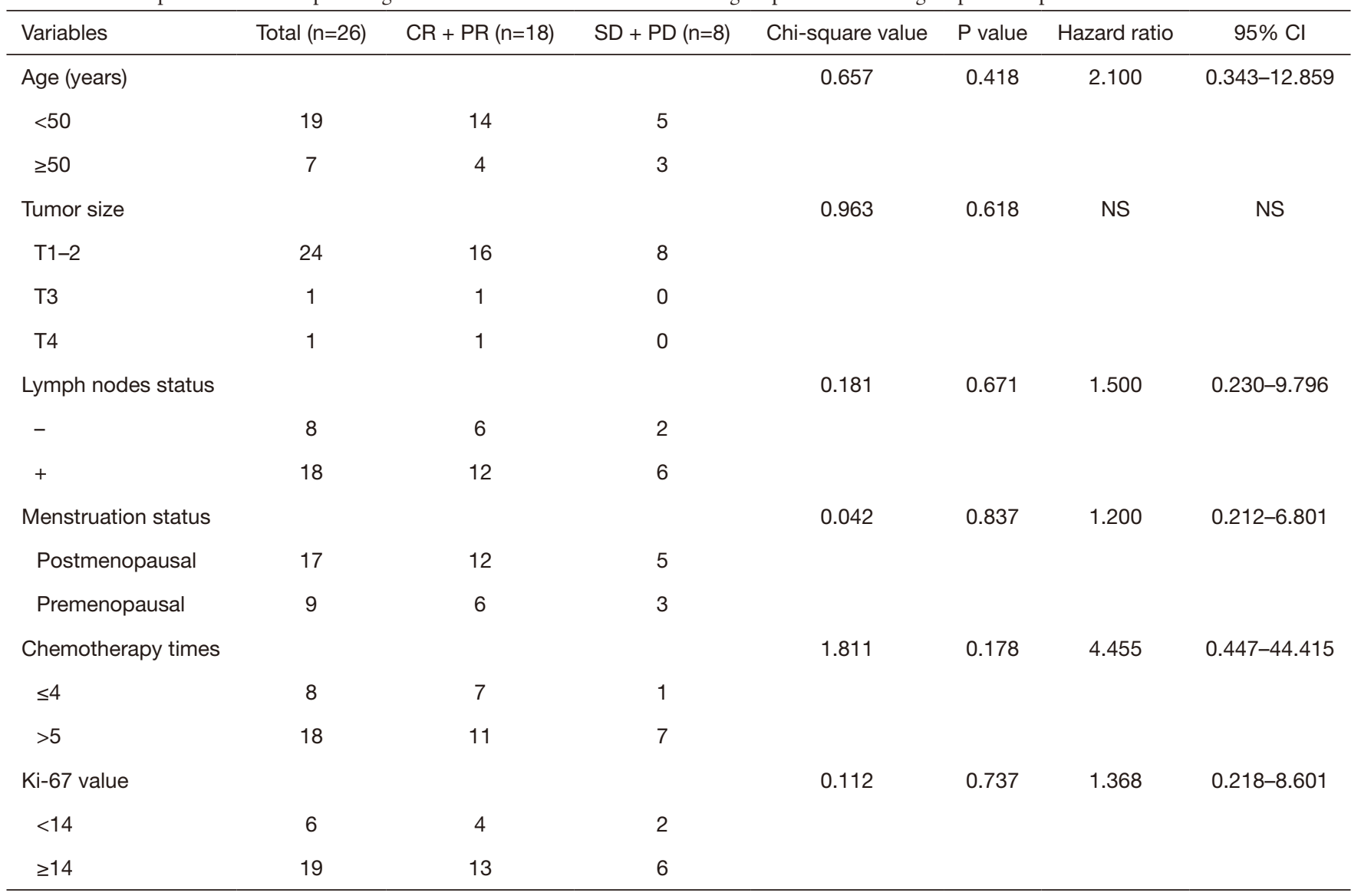

NS, no significance; CR, complete response; PR, partial response; SD, stable disease; PD, progressive disease. 